\title{
Efectos sísmicos en yacimientos arqueológicos: catalogación y cuantificación arqueosismológica
}

\author{
J.L. Giner-Robles ${ }^{(1)}$, M.A. Rodríguez-Pascua ${ }^{(2)}$, P.G. Silva ${ }^{(3)}$ y R. Pérez-López ${ }^{(2)}$ \\ (1) Departamento de Geología y Geoquímica, Facultad de Ciencias, Universidad Autónoma de Madrid, \\ Campus de Cantoblanco, s/n, E-28049 Madrid, España \\ jorge.giner@uam.es \\ (2) Instituto Geológico y Minero de España, Calle Ríos Rosas, 23. 28003 Madrid, España \\ ma.rodriguez@igme.es, r.perez@igme.es
}

(3) Departamento de Geología, Escuela Politécnica Superior de Ávila, Universidad Salamanca, E-05003 Ávila, España pgsilva@usal.es

\begin{abstract}
RESUMEN
El análisis de los daños producidos por eventos sísmicos en yacimientos arqueológicos proporciona mucha información sobre terremotos antiguos e históricos que pueden mejorar los análisis de peligrosidad sísmica. En este trabajo se propone una metodología de análisis que permite cuantificar la deformación a partir de los daños observados en un yacimiento de forma que se pueda establecer su posible origen sísmico. Esta metodología consta de tres fases: (a) Identificación e inventario de daños, (b) cuantificación de la deformación y (c) incorporación de los resultados a los catálogos sísmicos. La primera fase consiste en inventariar y cartografiar los daños siguiendo la clasificación de efectos arqueológicos de terremotos (EAE). La segunda fase consiste en cuantificar la deformación de esos daños, estableciendo diferentes parámetros como la dirección de máxima deformación horizontal (ey). Este análisis se realiza a diferentes escalas: individualmente para cada EAE inventariado, para el conjunto de daños del mismo tipo de efecto, y finalmente para todo el yacimiento considerado. El grado de homogeneidad en los resultados nos permite establecer el origen sísmico de las deformaciones. Esta metodología se ha desarrollado en al yacimiento romano de Baelo Claudia (Cádiz, España) y se ha aplicado a diferentes terremotos históricos de la Península Ibérica. Para calibrar este análisis, la metodología se ha aplicado a dos terremotos instrumentales: el terremoto de Lorca de 2011 (5,1 Mw; Murcia, España) y al terremoto de Emilia Romagna de 2012 (5,9 Mw; Italia).
\end{abstract}

Palabras clave: análisis estructural geológico, arqueosismología, direccionalidad de daños, efectos arqueológicos de terremotos (EAE).

\section{Seismic effects in archaeological sites: archaeoseismological cataloguing and quantification}

\begin{abstract}
The analysis of the damage caused by seismic events in archaeological sites provides significant information about ancient and historic earthquakes that can improve seismic hazard analysis. In this paper a methodological analysis to quantify the deformation of structural damage recorded in archaeological sites is proposed. This methodology focuses on the establishment of the seismic / non-seismic origin of the observed damage and consists of three phases: (a) identification and inventory of damage; (b) quantification of deformation; and (c) incorporation of the results to seismic catalogues. The first phase is the inventory and mapping of the damage according with the classification of archaeological effects of earthquakes (EAEs). The second phase is to quantify the deformation of damage by setting different parameters such as the direction of maximum horizontal strain (ey). This analysis is performed at different levels: for each of the recorded EAEs, for all the records of a particular category of EAEs, and finally for the complete archaeological site including all the categories of EAEs. The homogeneity of the results (damage orientation) allows us to establish the seismic origin of deformations. This methodology has been developed in the Roman site of Baelo Claudia (Cádiz, Spain) and has been applied to different historical earthquakes in the Iberian Peninsula. To calibrate the method, analyses on oriented damage were carried out immediately after the recent instrumental earthquakes of Lorca 2011 (5.1 Mw, Spain) and Emilia Romagna 2012 (5.9 Mw; Italy).
\end{abstract}


Giner-Robles, J.L., et al., 2018. Efectos sísmicos en yacimientos arqueológicos:... Boletín Geológico y Minero, 129 (1/2): $451-467$

Key words: archaeoseismology, earthquake archaeological effects (EAEs), oriented seismic damage, structural geological analysis.

\section{ABRIDGED ENGLISH VERSION}

\section{Introduction and methodology}

Archaeoseismology studies past earthquakes by assessing archaeological sites and contributing unpublished data on earthquakes that may not even have been historically recorded (Nikonov, 1988; Guidoboni, 1989; Stiros and Jones, 1996). Figure 1 clearly illustrates how archaeoseismology works as a link between classic historical records and geological records of earthquakes by merging both of them. Thus, we currently have several categories of earthquakes whose data may come from different types of analysis: instrumental earthquakes (i.e. registered by instruments); historical earthquakes (historically and archeologically documented); ancient earthquakes (archeologically and, in some cases, geologically documented); and paleoearthquakes (only geologically documented).

Therefore, archaeoseismology analyses ancient earthquakes and refines the data of some historical earthquakes using archaeological evidence of strain. Nevertheless, in many cases, these effects may have been caused by other phenomena, both of natural and anthropogenic origin. Usually, if there is no well-documented historical description of the event, it is difficult to assign a clear seismic origin to the damage observed.

The research described in this paper focuses primarily on establishing an analytical method to quantify deformation observed in archaeological sites, thereby enabling their possible seismic origin to be determined in a qualitative fashion

The methodology proposed in this paper consists of the following main stages (Fig. 2): a) damage identification and inventory, b) strain quantification and c) incorporating results in seismic catalogues

To identify damage, we have used the Earthquake Archaeological Effects classification method proposed by Rodriguez Pascua et al. (2011b) (Fig. 3), which distinguishes between co-seismic effects (geological effects and effects on building fabric) and post-seismic effects (Fig. 4). In this identification, archaeological and historical studies of the area should be taken into account, firstly to correctly interpret the structures and secondly to properly date them in order to be able to assign them to a particular earthquake. Many of the inventoried effects may have a multiple origin - this uncertainty can be eliminated using strain quantification analysis.

Strain quantification as part of the Earthquake Archaeological Effects (EAEs) is based on analysing those EAEs that are likely to provide data on the deformation process that produced or induced such effects; i.e. the co-seismic effects - both geological effects and the effects on building fabric.

This paper mainly develops an analysis of the effects on building fabric by applying different techniques used in structural geology. This fact allows us to directly compare the results obtained from analysing these structures with the results of the analysis of geological effects, given that the methodologies and therefore the results are similar.

The methodology used to analyse seismic effect-induced strain on building fabric at archaeological sites involves a different stages (Giner Robles et al., 2009) (Fig. 5).

Before analyzing the different deformations observed, several aspects regarding the data to be collected must be considered (Fig.6). These aspects focus on defining the parameters to be analysed to provide strain data and to ensure suitable characterization of the deformation kinematics (Fig.7).

Strain quantification on each analysed structure. Analysis of the tensors defined for each EAE (a single result for each kind of structure described at the site) so that the consistency of the data throughout the site depending on the type of structure can be analysed (Fig. 8). Overall site analysis, so that the homogeneity of the entire site can be assessed and where applicable, measured against the defined strain tensor.

With the results thus obtained, the degree of consistency in the results is evaluated, assigning it or not to a seismic origin. The definition of strain origin does not mean that inventoried effects can be assigned to a specific event. To assign such damage to a particular event, all structures must be properly dated and such datings have to be consistent with the event.

\section{Results and discussion}

This methodology has been developed in the Roman site of Baelo Claudia (Cádiz, Spain) and has been applied to different historical earthquakes in the Iberian Peninsula. At Baelo Claudia, earlier studies have defined the occurrence of two earthquakes with no historical reference in the period between 1 and 300 A.D. (Guidoboni, 1989; Sillières, 1997; Silva et al., 2005, 2009; Grützner et al., 2012). Such recognition was carried out by multidisciplinary collaboration between different experts (archaeologists, historians, geologists, architects, ...), col- 
Giner-Robles, J.L., et al., 2018. Efectos sísmicos en yacimientos arqueológicos:... Boletín Geológico y Minero, 129 (1/2): $451-467$

laboration that enabled the recognition of a range of damage and effects comparable to the occurrence of earthquakes close to the area, especially archaeological data (e. g. abandonment of parts of the city, the presence of destruction horizons).

The application and development of this method allowed the quantification of the deformation of the different effects observed in the reservoir (Fig. 9), establishing the presence of two destructive of earthquakes that affected the city in the I and III centuries, corroborating the previous hypotheses.

Within this framework, we are working on other archaeological sites to establish the possible occurrence of seismic events that are not catalogued, such as the possibility of a destructive event in the fourth century affect the Roman city of Complutum (Madrid, Spain) (Rodríguez-Pascua et al., 2016) (Fig.10).

To calibrate the methodology, analyses on oriented damage were carried out immediately after the recent instrumental earthquakes of Lorca 2011 (5.1 Mw, Spain) and Emilia Romagna 2012 (5.9 Mw; Italy).

The Lorca earthquake (Mw 5.3, Spain) of May 11, 2011, whose epicenter was located very close to the city (c.a. $3 \mathrm{~km}$ towards NW of the city), caused extensive damage and reached the macroseismic intensity of VII EMS. Giner-Robles et al. (2012) recorded almost 140 EAEs (Fig. 11). The different EAEs were distributed throughout the whole city, showing a main orientation according to $N 130^{\circ} \mathrm{E}$ and with very small dispersion. This orientation is almost orthogonal to the overall orientation of the Lorca-Alhama de Murcia Fault (NE-SW), the seismic source of the 2011 event, and also coincides with the orientation of the horizontal displacement vectors deduced from the ground acceleration data $(0.37 \mathrm{~g})$, which was recorded by the IGN (Instituto Geográfico Nacional) seismic station sited at the Lorca city centre.

The seismic sequence of Emilia Romagna (2012, Italy) of May 20 (Mw 5.9) also produced oriented damage, mostly being oriented wall collapses and falls (Fig. 12). The field analysis carried out for this zone included eleven localities around the epicentral area. The analysis of the orientation of the most frequent orientation of the deformation displayed two orthogonal directions $\left(\mathrm{N}_{330^{\circ}}\right.$ and $\left.\mathrm{N} 240^{\circ}\right)$ (Giner-Robles et al., 2011). These both orientations have been interpreted as either the occurrence of the two main earthquakes, or related to the orientation of the suspect seismic fault (Pérez-López et al., 2012).

The method proposed here provides a quantified analysis of archaeological sites likely to have been damaged by seismic events. These analyzes are based on the premise that the damage caused by an earthquake in a town (or archaeological site) follows a pattern of orientations related to the focal earthquake parameters (location, fault type, rupture directivity, etc.) (Giner-Robles et al, 2009, 2012; Howard et al., 2005; Motoki and Seo, 2000). There are many studies that have addressed the analysis of earthquake damage in archaeological sites and historic buildings (e. g. Guidoboni, 1989; Hancock and Stewart, 2001; Marco, 2008; Nikonov, 1988; Stiros and Jones, 1996). This methodology includes inventory (Rodriguez-Pascua et al., 2011b) and damage analysis using different characterization techniques, which are classically used in the strain analysis of structural geology (Giner-Robles et al., 2009, 2011). These techniques enable us to quantify the deformation at different scales so that we can establish, from patterns obtained damage orientation, homogeneity of the results, and therefore its possible seismic origin.

The method has been developed so that data can be compared (Rodríguez-Pascua et al., 2013) with the classic macroseismic intensities scales (EMS, MSK) and with intensity scales based on the geological effects of earthquakes (ESI-07, Michetti et al., 2007). Consequently, the data can be incorporated into the seismic catalogues and thus improve seismic hazard analysis.

\section{Introducción}

La arqueosismología permite inventariar y analizar los daños presentes en un yacimiento arqueológico o edificio histórico, de forma que se pueda establecer si las deformaciones presentes puedan tener un origen sísmico (Nikonov, 1988; Guidoboni, 1989; Stiros and Jones, 1996).

Este innovador análisis estudia terremotos del pasado mediante el análisis de yacimientos arqueológicos, aportando datos inéditos sobre sismos que pueden incluso no haberse registrado históricamente. La figura 1 ilustra claramente como la arqueosismología funciona como nexo de unión entre los registros históricos y los geológicos, intercalándose entre ellos.
Así, en la actualidad, podemos definir varias categorías de terremotos en función del tipo de datos utilizados en su análisis: (a) Terremotos instrumentales (registrados instrumentalmente); (b) Terremotos históricos (documentados histórica y arqueológicamente); (c) Terremotos antiguos (documentados arqueológicamente y en algunos casos geológicamente); y (d) Paleoterremotos (sólo documentados geológicamente).

Así pues la arqueosismología se encarga del análisis de terremotos antiguos y de la reinterpretación de datos de algunos terremotos históricos con evidencias arqueológicas de las deformaciones. No obstante, en muchos casos estos efectos pueden haberse producido por otro tipo de fenómenos, tanto de ori- 


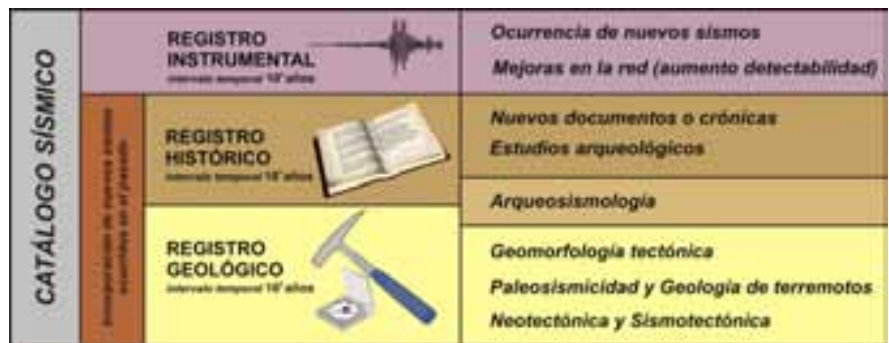

Figura 1. Existen disciplinas científicas que nos permiten analizar $y$ caracterizar eventos sísmicos que han ocurrido en el pasado y que son susceptibles de incorporarse al catálogo sísmico; bien desde el registro histórico, como con el estudio de nuevos documentos históricos, o con la incorporación de datos a partir de análisis arqueológicos que indiquen la ocurrencia de un sismo que haya afectado al yacimiento; o bien desde el registro geológico, con análisis geomorfológicos, o análisis paleosísmicos mediante la realización de trincheras que pueden permitir la caracterización de una falla activa a partir de la determinación de los eventos sísmicos que han quedado registrados en los materiales.

Figure 1. Different scientific disciplines enable us to analyze and characterize those seismic events that have occurred in the past and which are candidates to be included in the seismic catalogue. Either the historical record, such as the study of new historical documents or the incorporation of data from archaeological analysis, or the geological record, such as the geomorphological analysis obtained by digging paleoseismic trenches, give us information to complete the instrumental record of earthquakes and increase the temporal catalogue. gen natural como antrópico; y normalmente, sin una descripción histórica bien documentada, resulta complejo asignar un origen sísmico claro a los daños observados.

Este trabajo se centra fundamentalmente en presentar una metodología de análisis que permite cuantificar las deformaciones observadas en yacimientos arqueológicos, de forma que se pueda determinar cualitativamente su posible origen sísmico.

\section{Metodología de análisis arqueosismológico}

La metodología propuesta en este trabajo consta de tres partes principales (Fig. 2): (a) identificación e inventario de daños; (b) cuantificación de la deformación y datación; e (c) incorporación de los resultados a los catálogos sísmicos.

\section{Identificación e inventario de los daños}

El principal interés en la identificación e inventario de los daños es documentar y caracterizar todos los efectos susceptibles de haber sido producidos por terremotos destructivos en el intervalo histórico definido por el yacimiento arqueológico. En muchos

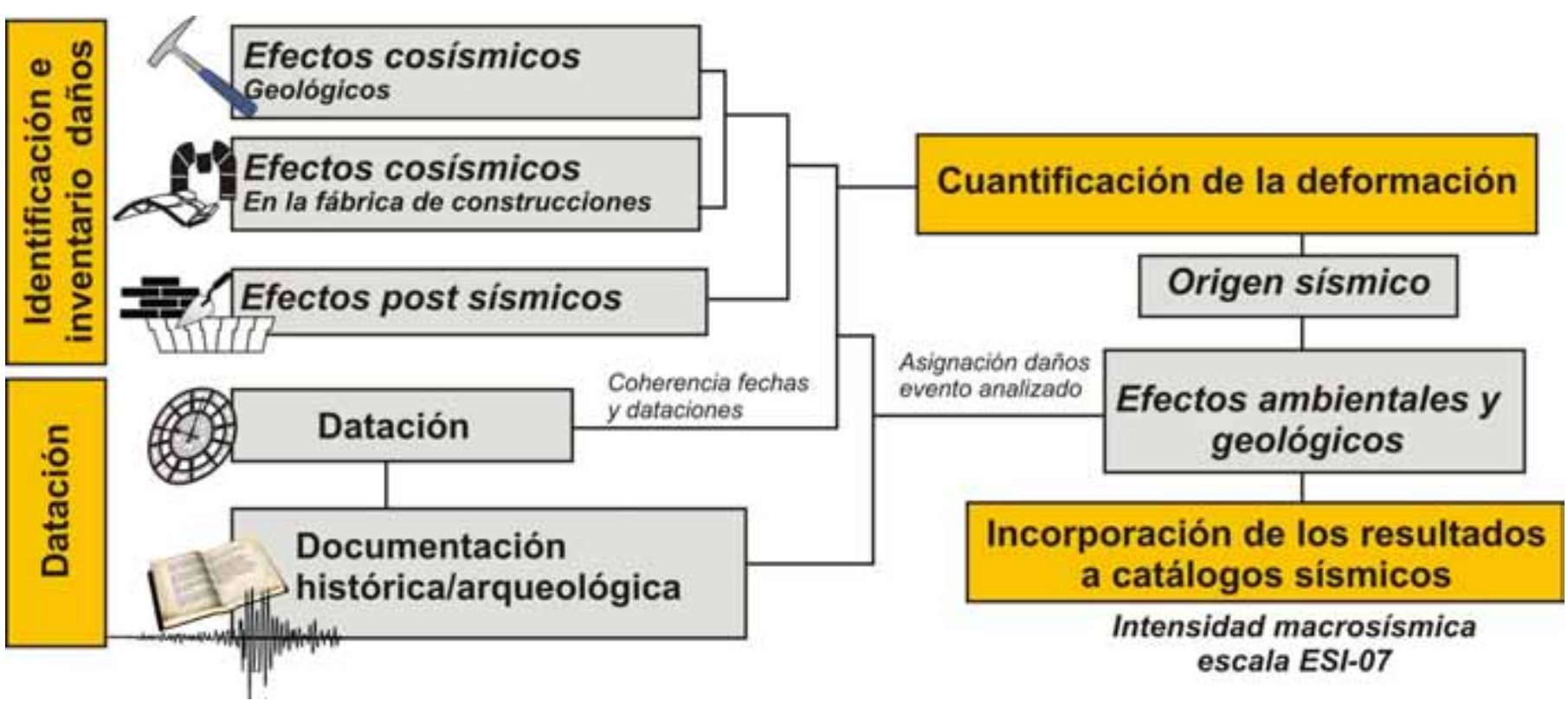

Figura 2. Esquema de la metodología propuesta en este trabajo: a) identificación de los daños, b) cuantificación de la deformación y datación, y c) incorporación de los resultados a los catálogos sísmicos.

Figure 2. Proposed methodology for archaeoseismic analysis: a) damage identification in buildings, b) strain quantification by different geological structural techniques, and c) incorporation of the results into seismic catalogues. 

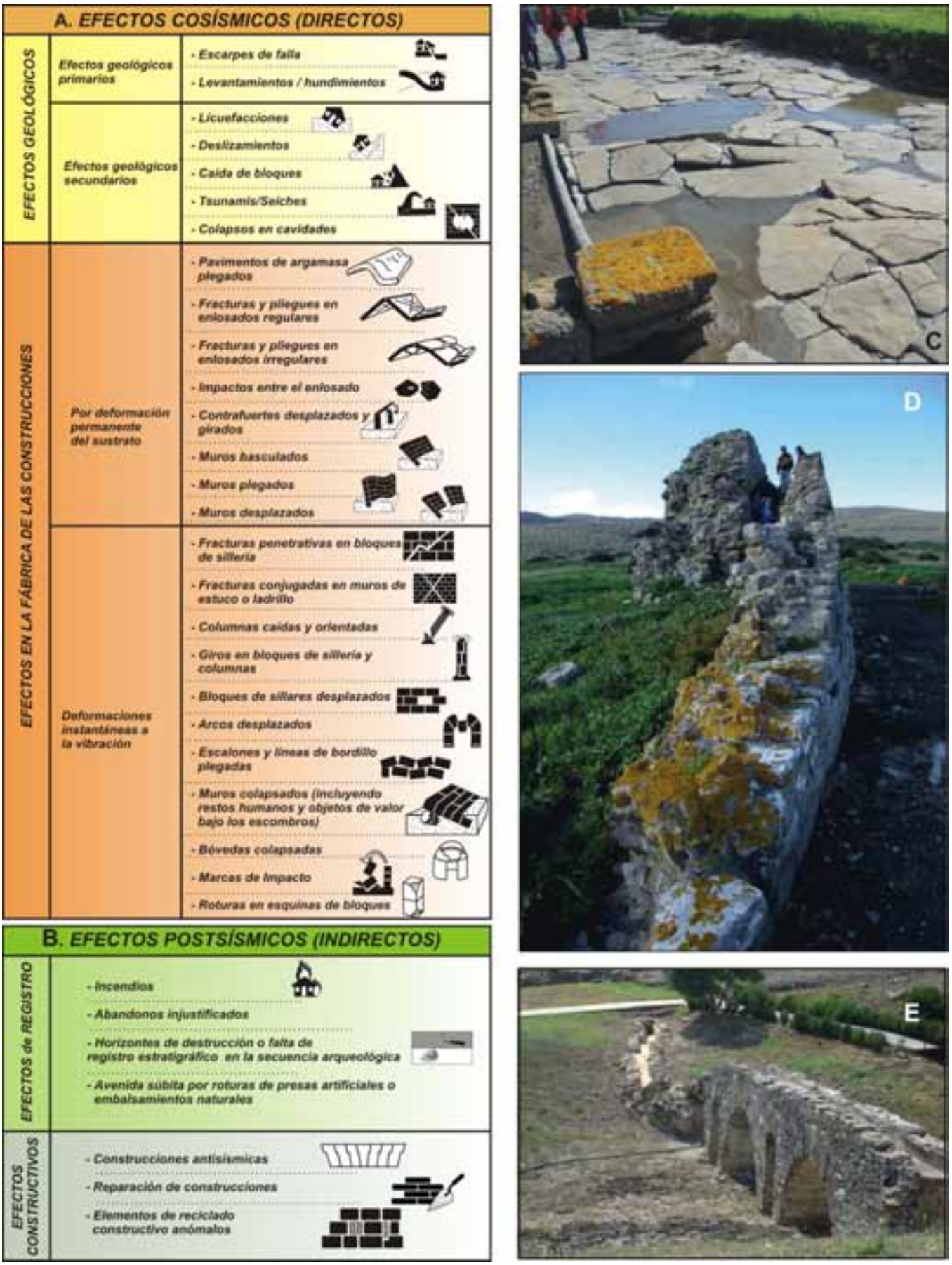

Figura 3. Tabla de clasificación de los Efectos Arqueológicos de Terremotos (Earthquake Archaeological Effects: EAE) (modificado de Rodríguez-Pascua et al., 2011b): a) efectos cosísmicos, efectos producidos directamente por el evento sísmico (geológicos y en la fábrica de las construcciones). b) efectos postsísmicos, efectos indirectos producidos por las consecuencias del terremoto, ya sean visibles en el registro geoarqueológico (efectos de registro), o en las construcciones posteriores al evento sísmico (efectos constructivos). c) Pliegues en el enlosado del Decumanus Maximus en el yacimiento romano de Baelo Claudia (Cádiz, España). d) Muro plegado en la muralla este de la ciudad de Baelo Claudia. e) Colapso y fracturación del acueducto este de la ciudad de Baelo Claudia (todos los daños fueron producidos por un terremoto en el siglo IV AD, Silva et al., 2014).

Figure 3. Classification table of Earthquake Archaeological Effects (EAEs) (modified from Rodríguez-Pascua et al., 2011b): a) co-seismic effects, effects directly caused by the seismic event. b) Post-seismic effects, indirect effects caused by the consequences of the earthquake, whether visible in the geo-archaeological record (registered effects), or in edifices after the seismic event (construction effects). c) Folds in the pavement of the Decumanus Maximus at the Roman site of Baelo Claudia (Cádiz, Spain. d) Wall folding on the east wall of the city of Baelo Claudia. e) Collapse and fracturing of the aqueduct in the city (Baelo Claudia). (All the damage was caused by an earthquake in the IVth century $A D$ ) (Silva et al., 2014). 


\section{otros periodos y culturas}

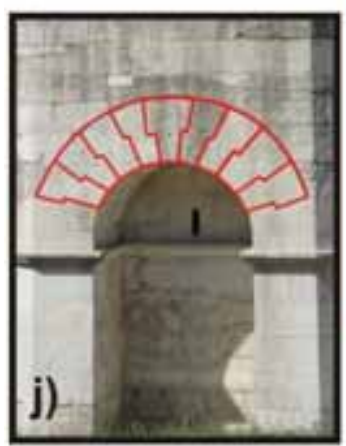

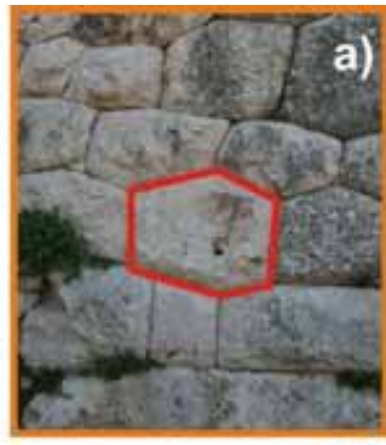
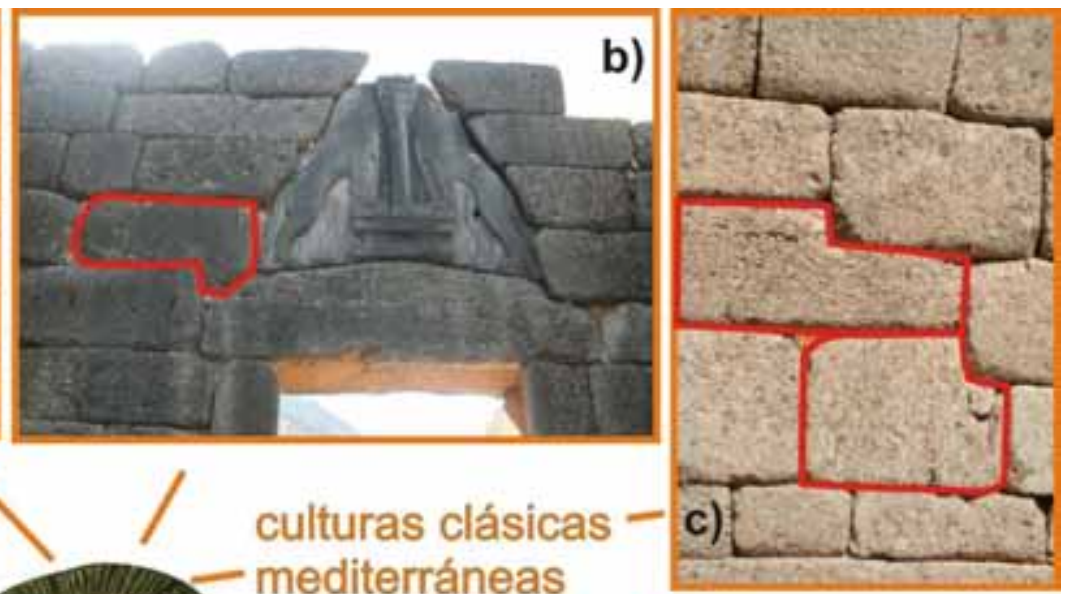

periodos islámico

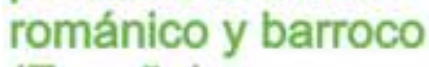
(España)
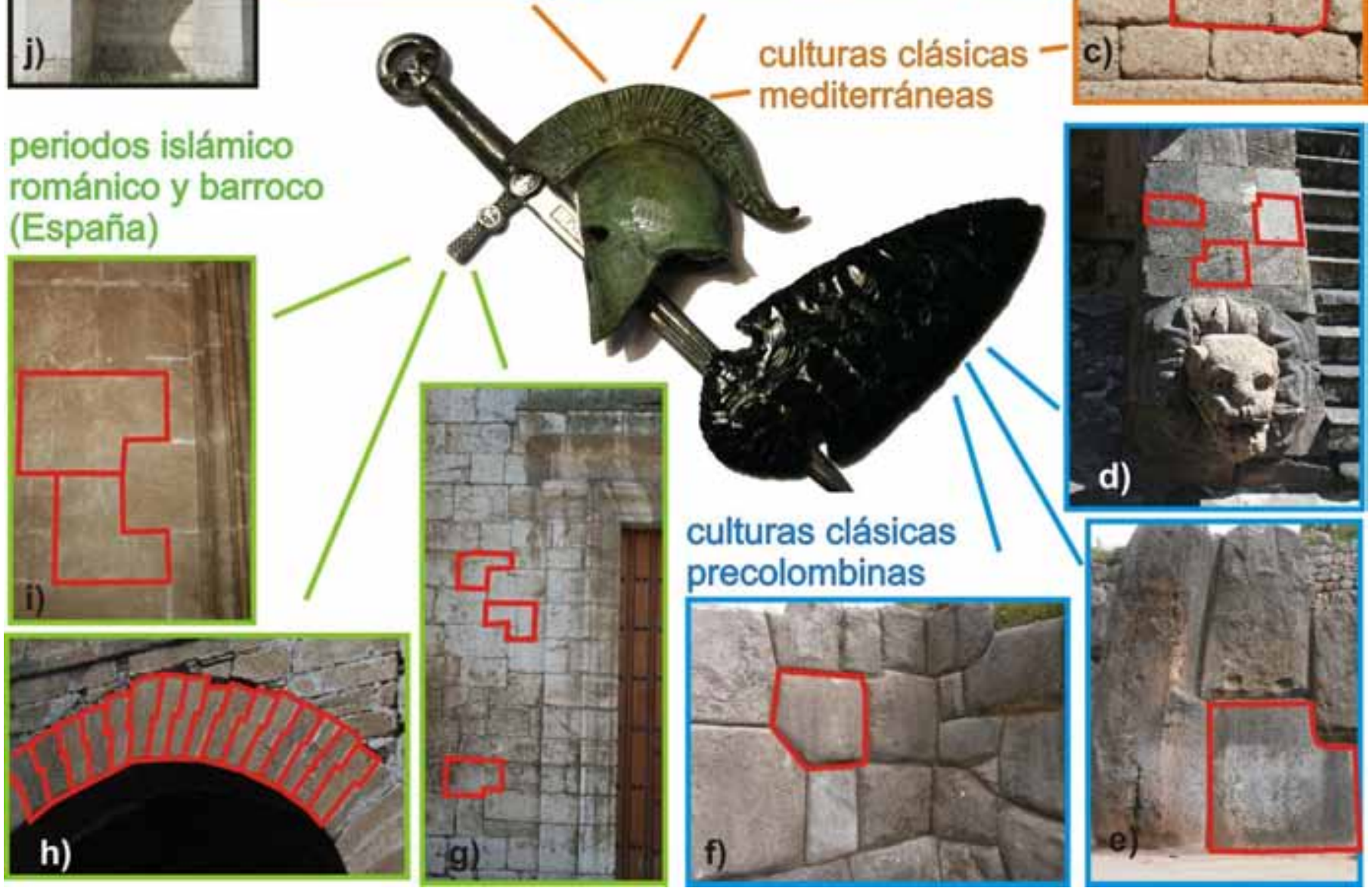

Figura 4. Una de las medidas de construcción sismoresistente más extendida es el tallado y ajuste de bloques de forma que se rompe la hilada horizontal en las construcciones. Esta asimetría le confiere a la estructura una mejor respuesta frente a los movimientos sísmicos. Existen ejemplos de esta técnica constructiva en diferentes culturas y periodos. En culturas mediterráneas clásicas como en Micenas (Grecia): en la muralla ciclópea de la ciudad (a), en la Puerta de los Leones (b), o en la entrada de la Tumba de Agamenon (c), o en periodos culturales más modernos como el Mausoleo deTeodorico (j) en Rávena (Italia). También se observan en culturas precolombinas, como en las pirámides de Teotihuacan (México) (d), o en las construcciones incas de Cuzco (Perú) (e y f). Este tipo de estructuras también se observa en diferentes periodos culturales en España: en construcciones barrocas como la Lonja de Valencia ( $\mathrm{g}$, $\mathrm{i}$ ), y en construcciones del periodo islámico como la Puerta de Sevilla en Carmona (h) (Sevilla).

Figure 4. One of the most popular techniques for earthquake resistant constructions is the use of sculpted and setting blocks. The asymmetry of these blocks gives the structure a better response to earthquake vibration. There are lots of examples of this technique in different cultures and periods around the world. For example, during the classical Mediterranean culture as in Mycenae (Greece): the cyclopean city wall (a), the Lions Gate (b), or at the entrance to the Tomb of Agamemnon (c), also the more recent period as the Mausoleum of Theodoric (j) Ravenna (Italy). They are also observed in the pre-Columbian cultures such as the pyramids of Teotihuacan (d) (Mexico), or the Inca constructions of Cuzco (Peru) (e and f). This type of structure is also present in Spain: the baroque buildings as in the "Lonja de Valencia" $(g, i)$, and constructions of the Islamic period such as the "Puerta de Sevilla" in Carmona (h) (Sevilla) for instance. 
casos, tenemos constancia histórica de los efectos (e. g. terremoto de Lisboa de 1755) (Martínez-Solares, 2001), pero en otros casos no existe documentación que certifique la ocurrencia de un terremoto, como por ejemplo los terremotos que afectaron entre los siglos I y III AD a la ciudad romana de Baelo Claudia (Cádiz) (Silva et al., 2014a). En estos casos es imprescindible analizar y recopilar toda la información del emplazamiento, e identificar correctamente los efectos susceptibles de ser analizados, también es necesaria la consulta tanto de los informes de las excavaciones realizadas en el emplazamiento, como de la documentación existente sobre trabajos de restauración y consolidación arqueológica.

Para este inventario utilizaremos la clasificación de efectos arqueológicos de terremotos (EAE) propuesta por Rodríguez-Pascua et al. (2011b) (Fig. 3). Los efectos arqueológicos de terremotos son todos aquellos efectos asociados a la ocurrencia de un sismo y que se pueden analizar a partir del registro arqueológico y geológico dentro del contexto de un yacimiento arqueológico.

\section{Cuantificación de la deformación}

Es evidente que muchos de los efectos referidos (Fig. 3) pueden producirse por diferentes procesos naturales y/o antrópicos. No obstante hay que tener en cuenta que los evento sísmicos pueden producir daños orientados, es decir los daños pueden presentar direcciones predominantes relacionadas con los parámetros focales del terremoto (localización epicentral, orientación y tipo de falla, directividad de la rotura, etc...) (e. g. Howard et al., 2005; Motoki and Seo, 2000). El objetivo de cuantificar la deformación presente en un yacimiento es determinar el grado de homogeneidad en la orientación de daños: si las direcciones de daño obtenidas presentan orientaciones homogéneas podemos deducir que el conjunto de los daños está producido por un evento sísmico, en cambio si las deformaciones presentan orientaciones muy dispersas y heterogéneas, no podremos establecer el origen común de las deformaciones, y por tanto asimilarlas a un evento sísmico.

Por lo tanto, una vez identificados e inventariados los diferentes $E A E$, se debe cuantificar la deformación presente en los efectos cosísmicos observados (Fig. 3a), tanto en los efectos geológicos, como en los efectos en la fábrica de las construcciones.

En este trabajo se desarrolla fundamentalmente el análisis de los efectos en la fábrica de las construcciones, aplicando diferentes técnicas utilizadas en geología estructural. Esto nos permite comparar directamente los resultados obtenidos del análisis de estas estructuras con los resultados obtenidos del los efectos geológicos, ya que las metodologías, y por tanto los resultados, son análogos. Los efectos postsísmicos (Fig. 3b) pueden ser utilizados para apoyar las conclusiones o aportar mayor definición a las dataciones de los efectos cosísmicos. Uno de los más comunes es la utilización de medidas de construcción sismoresistente (Fig. 4). Aunque también es impor-

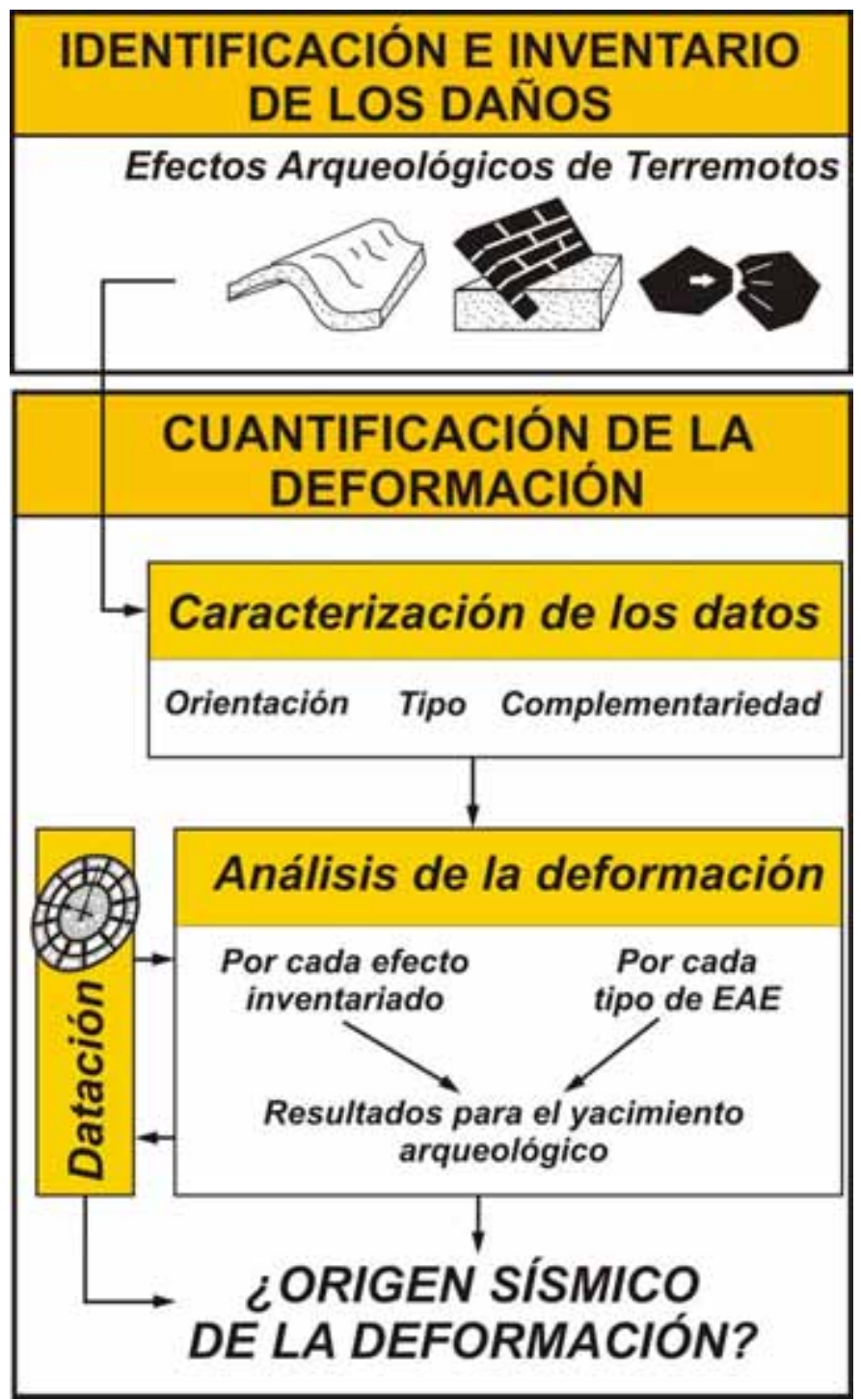

Figura 5. Esquema metodológico propuesto por Giner-Robles et al. (2009), para el análisis cuantitativo de las deformaciones presentes en estructuras de un yacimiento arqueológico (EAE) (Rodríguez Pascua et al., 2011b).

Figure 5. Methodological diagram proposed by Giner-Robles et al. (2009) for the quantitative analysis of the strain deformation affecting buildings on the archaeological site (EAE) (Rodriguez Pascua et al., 2011b). 
tante resaltar que algunas de estas técnicas constructivas también se utilizan en reparaciones y ampliaciones de estructuras, por lo que es necesario analizar adecuadamente este tipo de estructuras para poder asignarles una clara función antisísmica (GinerRobles et al., 2011 y 2012b).
La metodología aplicada al análisis de la deformación de los efectos sísmicos en la fábrica de las edificaciones en yacimientos arqueológicos consta de una serie de fases (Giner Robles et al., 2009) (Fig. 5): caracterización de los datos, análisis de la deformación y datación.
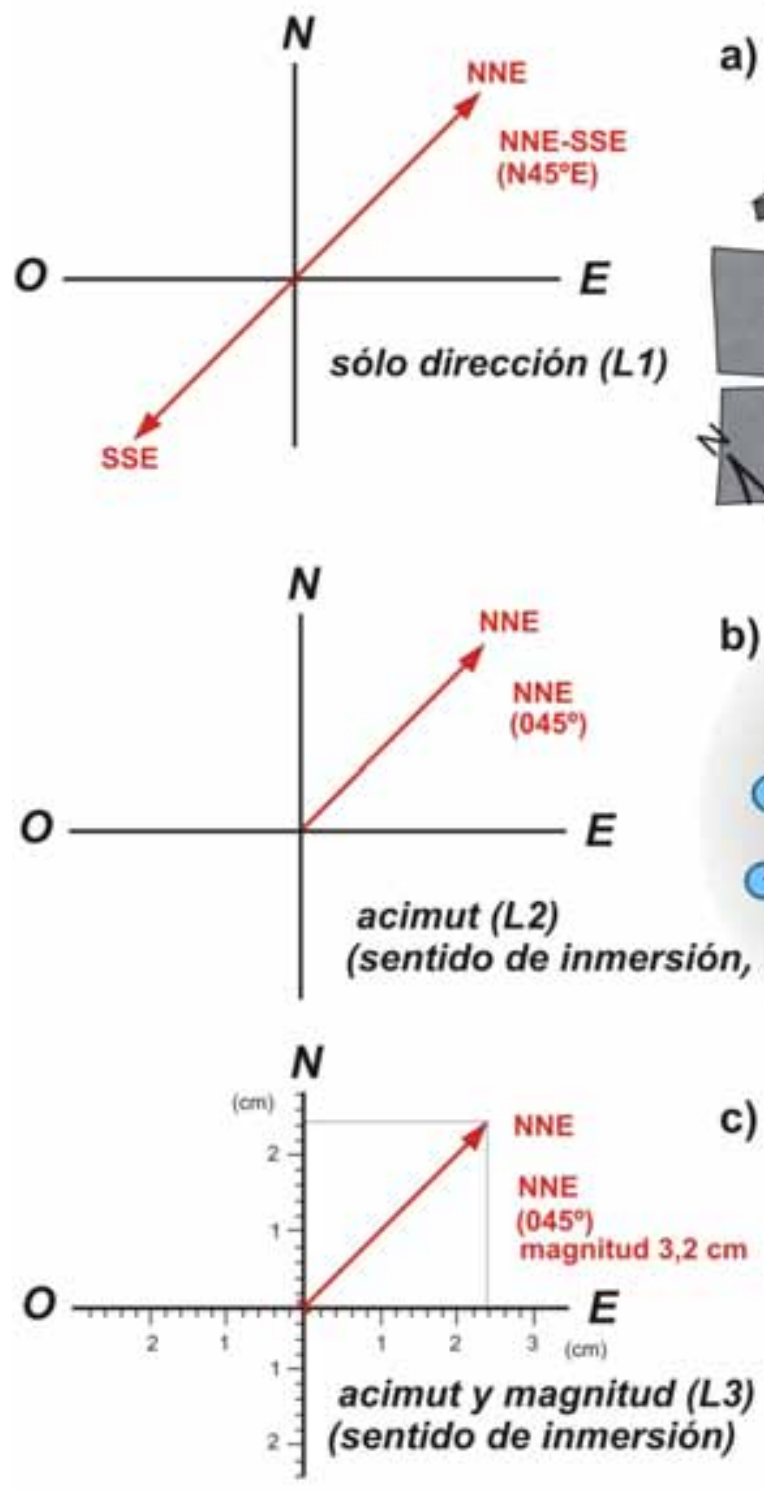

c)

\section{PLIEGUES EN ENLOSADOS}
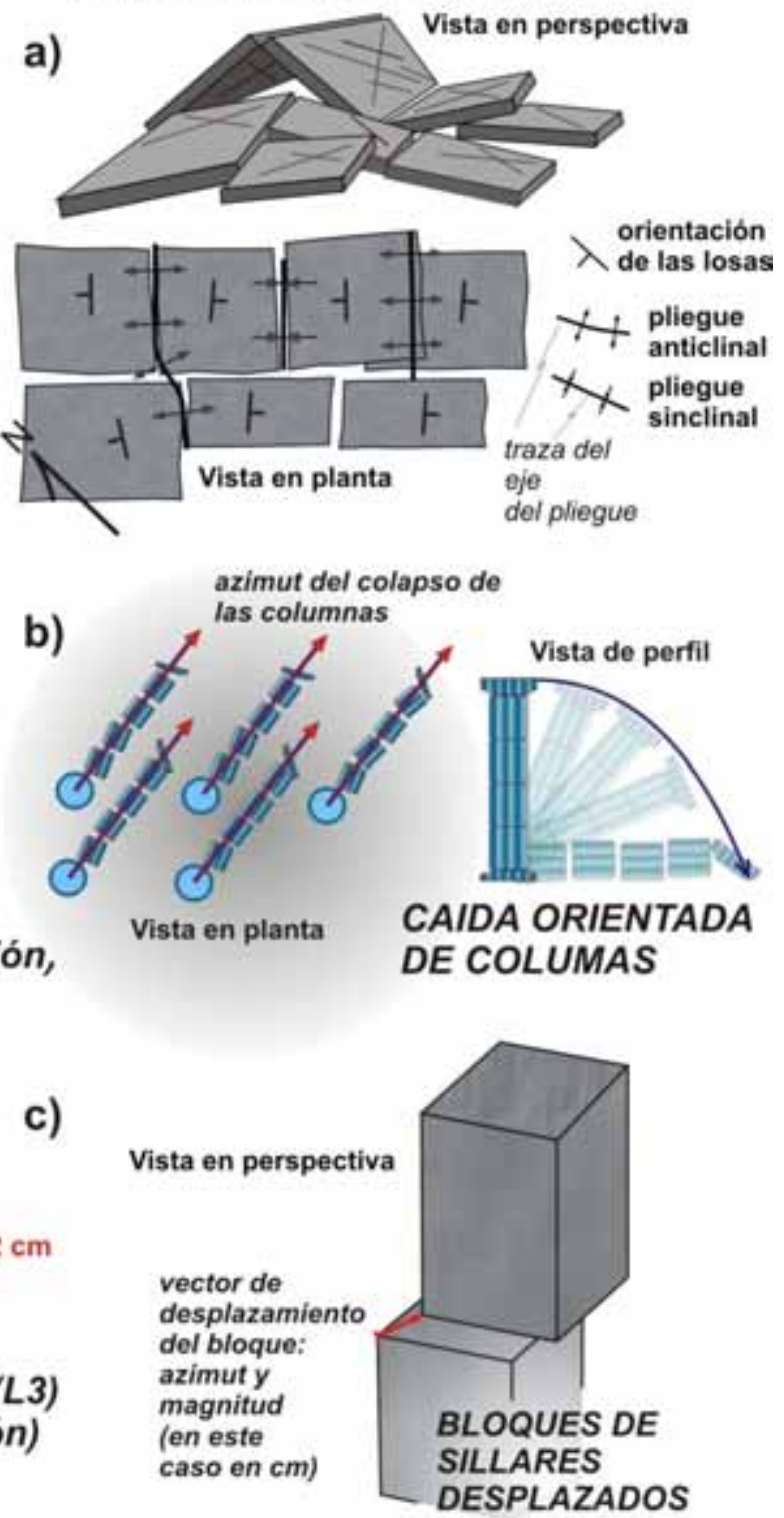

Figura 6. Caracterización de los datos lineales que se deben analizar en los yacimientos arqueológicos en función de su orientación: a) L1, sólo se puede definir la dirección de máximo acortamiento horizontal (ey); b) L2, los efectos tienen una direccionalidad marcada, además de poder establecer su dirección, podemos establecer el sentido de los daños; c) L3, podemos obtener los mismos datos (azimut y sentido) que en los datos de tipo L2, pero además podemos cuantificar la magnitud de esa deformación (e. g. vectores de desplazamiento de bloques: azimut, sentido de movimiento, y magnitud de ese desplazamiento).

Figure 6. Characterization of the linear data to be analyzed based on their orientation: a) L1-type, only the direction of the maximum horizontal shortening (ey) can be established, b) L2-type, in this case the observed effects have directionality and it is possible to determine the sense of the damage; c) L3-type, as in the previous case, the azimuth and the direction can be determined, and the magnitude of the deformation can also be quantified (e. g. block displacement vectors: azimuth, sense of motion, and magnitude of that displacement). 


\section{Caracterización de los datos.}

Antes de analizar las diferentes deformaciones observadas, debemos considerar una serie de aspectos relacionados con los datos que se deben recopilar. Estos aspectos se centran en la definición de los parámetros analizables para la caracterización adecuada de la cinemática de las deformaciones y por tanto del tensor de deformación. Los parámetros considerados son: (a) orientación, (b) tipo y (c) grado de complementariedad (Giner-Robles et al., 2011) (Fig. 6).

(a) Orientación. Las orientaciones que debemos considerar para poder realizar el análisis estructural de los EAE en el yacimiento pueden presentar dos geometrías: (a1) planos, datos definidos mediante superficies (dirección y buzamiento) (e. g. superficies plegadas, $u$ orientaciones de losas en pavimentos plegados); y (a2) líneas, datos definidos por una orientación de una línea, y que pueden ser de tres tipos: sólo dirección (L1) (e. g. E-O, N45ํㅡ, ...); dirección y azimut (e.g. vector de caída de columnas) (L2), es decir, definimos el sentido de una dirección (conocemos la dirección de la línea y su sentido); y por último cuando conocemos la dirección, el azimut y la magnitud (e. g. vectores de desplazamiento) (L3).

(b) Tipo de dato. Los datos recopilados pueden definir bien una orientación única de un plano o de una línea (e.g. choques entre losas de pavimentos); o bien un intervalo de orientaciones (e.g. basculamientos de muros).

(c) Complementariedad de los datos. Pueden ser datos únicos en los que el EAE analizado aporta una única orientación o intervalo de orientaciones que no se pueden acotar comparando con otros datos obtenidos del análisis del mismo elemento en otras zonas del yacimiento arqueológico. $O$ bien pueden ser datos complementarios (e.g. muros, dovelas hundidas en arcos), cuyo análisis conjunto en el yacimiento permite acotar intervalos y orientaciones mediante la consideración de elementos similares en el tratamiento conjunto de los datos.

Es importante establecer en el inventario de los datos una cartografía que nos permita su representación en la planimetría del yacimiento para su posterior análisis (Giner-Robles et al., 2011, Silva et al., 2016) (Fig.7). Esta representación nos permite obtener directamente de la cartografía los datos necesarios para realzar el análisis de la deformación.

\section{Análisis de la deformación}

El análisis de las orientaciones de los daños nos permite establecer la orientación de máxima deforma- ción (ey) que los ha producido. Esta orientación se calcula específicamente para cada uno de los tipos de EAE analizados. Giner-Robles et al $(2009 ; 2011)$ desarrollaron el análisis pormenorizado de cada una de los principales EAE (Fig. 8).

Una vez obtenidos las orientaciones individuales de ey, debemos realizar análisis de los datos de forma conjunta. El análisis de los resultados definidos para cada uno de los tipos de EAE (un solo resultado para cada tipo de estructura), de forma que se pueda analizar la coherencia de los datos en todo el yacimiento en función del tipo de estructura. Y finalmente el análisis conjunto de todos los datos obtenidos, de forma que se pueda evaluar, y en su caso establecer, la homogeneidad de todo el yacimiento frente a las orientaciones principales de daño definidas.

Los análisis conjuntos de los datos tanto a nivel EAE como a escala del todo el yacimiento, son necesarios para poder minimizar los efectos locales, efectos que pueden estar relacionados tanto con las mismas estructuras analizadas (como la orientación de calles o de estructuras murarías), como con la presencia de otras estructuras que puedan llegar a modificar las orientaciones de máxima deformación (canalizaciones subterráneas, antiguas cimentaciones, etc.) (Giner-Robles et al., 2009; 2011).

\section{Datación}

Con las orientaciones obtenidas, se evalúa el grado de coherencia de los resultados, que permita asignar o no el origen sísmico de las deformaciones. Para poder asignar esos daños a un evento concreto, las edades de las estructuras deformadas deben ser coherentes entre si, ya que la definición del origen sísmico de las deformaciones no implica necesariamente que los efectos inventariados puedan ser asignados a un sólo evento, es decir se pueden localizar en el yacimiento daños producidos por varios eventos en diferentes fechas. Además también es importante analizar adecuadamente el registro arqueológico para evitar confusiones entre la fecha de construcción y la del daño producido por el terremoto.

\section{Incorporación de los resultados a los catálogos sísmicos}

Una vez definido el origen sísmico de las deformaciones y asignados los daños a un evento concreto, se debe asignar una intensidad macrosísmica al evento para que pueda ser incorporado a los catálogos sís- 


\begin{tabular}{|c|c|c|c|}
\hline \multicolumn{3}{|c|}{ a. EFECTOS COSISMICOS (DIRECTOS) } & Simbología cartográfica y de análisis \\
\hline \multirow{6}{*}{ 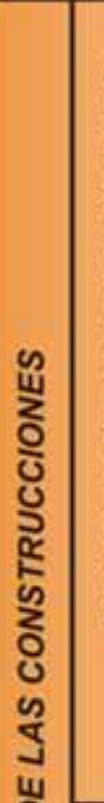 } & \multirow{6}{*}{ 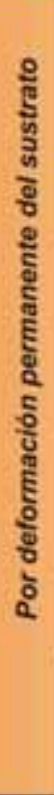 } & $\begin{array}{l}\text { - Pavimentos de argamasa } \\
\text { plegados }(P, L 1 / O / U) \\
\text { - Fracturas y pliegues en } \\
\text { enlosados rogulares } \\
(P, L 1, L 2 / O / U) \\
\text { - Fracturas y pliegues en } \\
\text { enlosados irregulares } \\
(P, L 1, L 2 / O / U)\end{array}$ & pop-up el valor numérico marca el acimut \\
\hline & & $\begin{array}{l}\text { - Impactos entre el } \\
\text { enlosado }(L 2 / O / U)\end{array}$ & $\begin{array}{l}\text { el valor numerico marca el acimut del impacto } \\
\text { entre las losas, paralelo a la dirección de ey }\end{array}$ \\
\hline & & $\begin{array}{l}\text { Contrafuertes desplazados } \\
\text { y girados }(L 3 / O / U)\end{array}$ & $\begin{array}{l}\text { ntación sobre la cual se } \\
\text { splazamiento, la flecha } \\
\text { tivo del movimiento. }\end{array}$ \\
\hline & & $\begin{array}{l}\text { - Muros basculados } \\
\text { (L2, L3/R/C) }\end{array}$ & 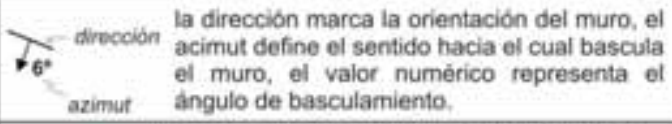 \\
\hline & & $\begin{array}{l}\text { - Muros desplazados } \\
(P, L 3 / O / U)\end{array}$ & $\begin{array}{l}\text { la linea define la orientación sobre la cual se } \\
\text { ha producido el desplazamiento, la flecha } \\
\text { marca el sentido relativo del movimiento. }\end{array}$ \\
\hline & & $\begin{array}{l}\text { - Muros Plegados } \\
(P, L 1 / O / U)\end{array}$ & $\begin{array}{l}\text { la traza del pliegue } \\
\text { marca la orientación } \\
\text { del eje }\end{array}$ \\
\hline$\frac{\pi}{\sqrt{2}}$ & \multirow{10}{*}{ 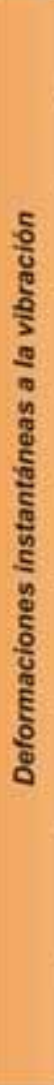 } & $\begin{array}{l}\text { - Fracturas penetrativas } \\
\text { en bloques de silleria } \\
(P / O / U)\end{array}$ & \\
\hline$\frac{m}{4}$ & & $\begin{array}{l}\text { - Fracturas conjugadas en } \\
\text { muros de estuco o ladrillo } \\
(\mathrm{P} / \mathrm{O} / \mathrm{U})\end{array}$ & on la dirección marca la orientación del muro \\
\hline$\sum_{i=1}^{5}$ & & $\begin{array}{l}\text { - Columnas caidas y orientadas } \\
(\mathrm{L} 2 / \mathrm{O} / \mathrm{U})\end{array}$ & $\begin{array}{c}\text { azimut la linea define el acimut de caída de la } \\
\text { columna, se puede poner también el valor } \\
\text { numerico }\end{array}$ \\
\hline$\stackrel{5}{0}$ & & $\begin{array}{l}\text { - Giros en bloques de silleria y } \\
\text { columnas }(L 3 / O / U)\end{array}$ & $\begin{array}{c}\text { giro en el sentido de } \\
\text { las agujas del } \\
\text { reloj (dextro) }\end{array}\left(\begin{array}{c}\text { giro en sentido contrario } \\
\text { a las agujas del } \\
\text { reloj (siniestro) }\end{array}\right.$ \\
\hline 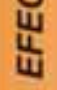 & & $\begin{array}{l}\text { - Bloques de sillares } \\
\text { desplazados }(\mathrm{L3} / \mathrm{O} / \mathrm{U})\end{array}$ & $\begin{array}{l}\text { 9l vector marca el sentido de movimiento del } \\
\text { bloque más alejado del suelo, el valor numerico } \\
\text { representa el desplazamiento en cm }\end{array}$ \\
\hline & & $\begin{array}{l}\text { - Arcos desplazados } \\
\text { (L1, L2/R/C) }\end{array}$ & $\begin{array}{l}\text { vimiento vertical de dovelas } \\
\text { vimiento horizontall de dovelas }\end{array}$ \\
\hline & & $\begin{array}{l}\text { Escalones y lineas } \\
\text { de bordillo plegadas } \\
(L 1 / O / U)\end{array}$ & 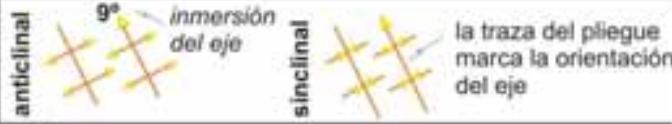 \\
\hline & & $\begin{array}{l}\text { - Muros colapsados } \\
(L 2 / R / C)\end{array}$ & $\begin{array}{l}\text { azimut la dirección marca la orientación del muro, el } \\
\text { dirocción el muro }\end{array}$ \\
\hline & & $\begin{array}{l}\text { - Marcas de Impacto } \\
(L 2 / R / U)\end{array}$ & $\begin{array}{l}\text { irección la direcolón marca la orientación del muro } \\
\text { del que se supone que ha caido el bloque } \\
\text { que define el impacto, en el caso de } \\
\text { conocerlo }\end{array}$ \\
\hline & & $\begin{array}{l}\text { - Roturas en esquinas de bloques } \\
\text { (L2/O/U) }\end{array}$ & $\begin{array}{l}\mathbf{A}^{45 / 255} \text { zona superior } \\
\text { bloque } \\
\nabla^{45 / 255} \begin{array}{l}\text { zona inferior de valor numérico define el sentido } \\
\text { bloque }\end{array}\end{array}$ \\
\hline
\end{tabular}

Figura 7. Simbología cartográfica y de análisis para los principales EAE que se pueden analizar en un yacimiento arqueológico (Silva et al., 2015). Se incluye también la caracterización de los parámetros considerados en el análisis de la deformación en cada EAE. Orientación del dato: planos (P) y líneas (L1, L2 y L3). Tipo de dato: orientación única (O) y rango de orientaciones (R). Y complementariedad del dato: dato único (U) y dato complementario(C) (ver texto para explicación).

Figure 7. Cartographic symbology for major EAEs in an archaeological site (Silva et al., in press). The characterization of the parameters considered in the strain analysis of EAE has also been included. Data orientation: planes ( $P$ ) and lines (L1, L2 and L3). Data type: single orientation $(O)$ and range of orientations $(R)$. Data complementarity: single data $(U)$ and complementary data $(C)$ (see text for further explanation). 

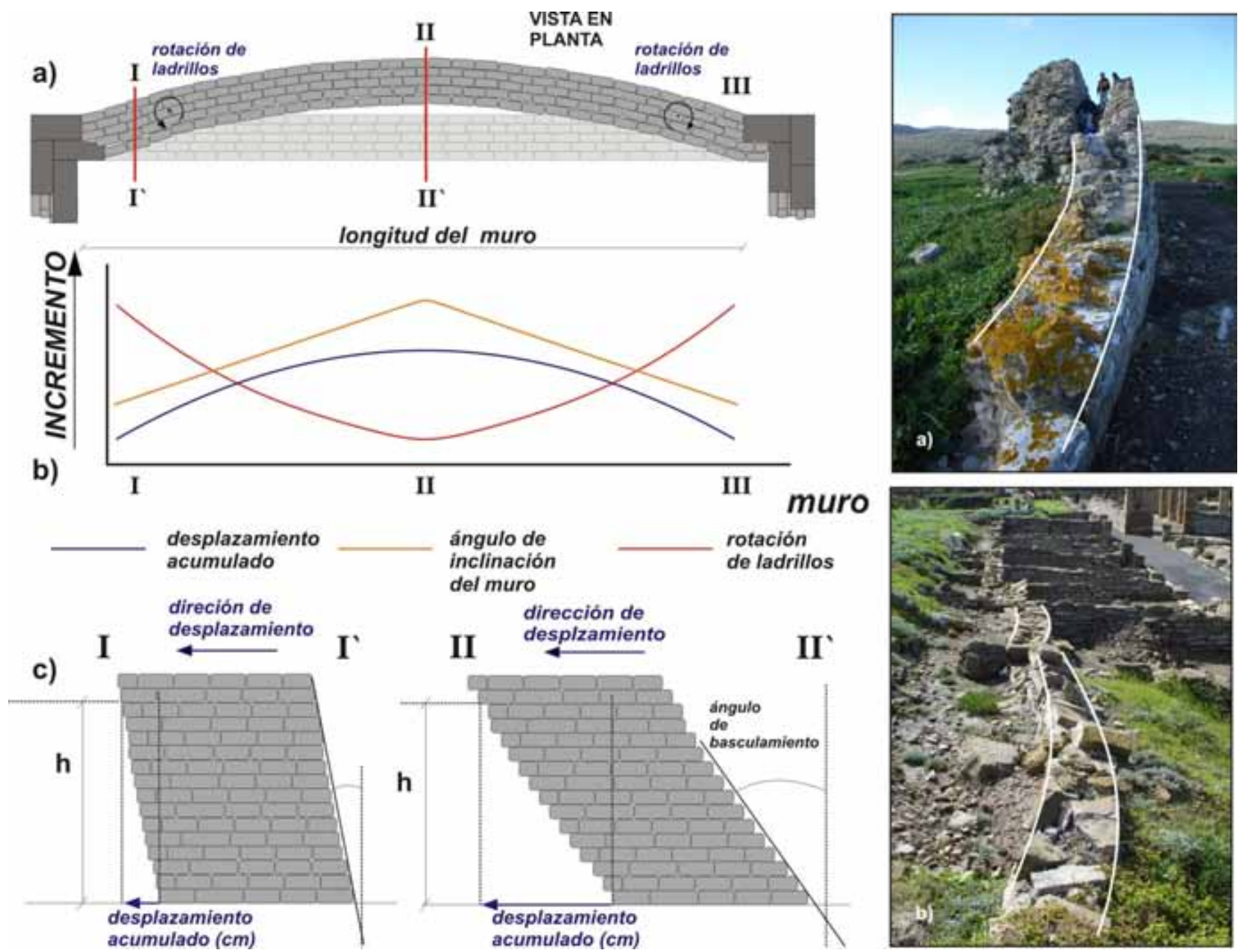

Figura 8. Ejemplo de análisis de uno de los EAE (muros plegados). Efectos de la deformación perpendicular al plano de un muro de ladrillo o de pequeños sillares irregulares anclado en sus extremos a estructuras más rígidas. a) Vista en planta de las deformaciones presentes (e. g. desplazamientos, rotaciones). b) Gráfico en el que se representan en función de la posición con respecto a los extremos anclados del muro las intensidades del desplazamiento del muro, el ángulo de basculamiento y la rotación de ladrillos o sillares. c) Vista de perfil de las zonas externas del muro (posición I-I, zona anclada), y de la zona central (II-II ), zona con mayor grado de libertad de movimientos.

Figure 8. Example of the analysis of EAE (namely folded walls). Effects of the strain perpendicular to the plane of a brick or small irregular masonry walls anchored at their ends to rigid structures. a) Plan view of the visible deformation (e. g. displacement, rotation). b) Graph which plots the wall displacement intensity, tilt angle and brick or stone rotation against the position with regard to the anchored wall ends. c) Side view of the outer parts of the wall (position II', anchored area) and central portion (II-II'), the area with greater freedom of movement.

micos utilizados en los análisis de peligrosidad sísmica.

Para ello utilizamos la escala macrosísmica de intensidades ESI-07 (Michetti et al., 2007), que utiliza los efectos ambientales y geológicos producidos por los terremotos para determinar una escala de intensidades comparable a las escalas clásicas más utilizadas (EMS, MSK, etc...).
Rodríguez-Pascua et al. (2013) establecen una correlación preliminar entre los EAE y la intensidad ESI-07. Recientemente se ha publicado el primer catálogo de los efectos geológicos de los terremotos en España (Silva et al., 2014a), en el que se revisan diferentes terremotos históricos a partir de los EEE y los $E A E$, de forma que se puedan incorporar los resultados al análisis de la peligrosidad símica en España. 


\section{Aplicación de la metodología}

La metodología propuesta se ha aplicado a diferentes terremotos antiguos e históricos. El principal enclave analizado ha sido el yacimiento romano de Baelo Claudia (Cádiz, España), en el que se desarrolló y estructuró la metodología de análisis propuesta en este trabajo (Fig. 9), corroborando a la vez las hipótesis previas de la ocurrencia de dos sismos que afectaron a la ciudad en los siglos I y III (Sillières, 1997; Silva et al., 2005; 2009; Grützner et al., 2012).

Estos sismos no estaban incluidos en los catálogos sísmicos previos de la Península lbérica, lo que confirma que este tipo de análisis son adecuados

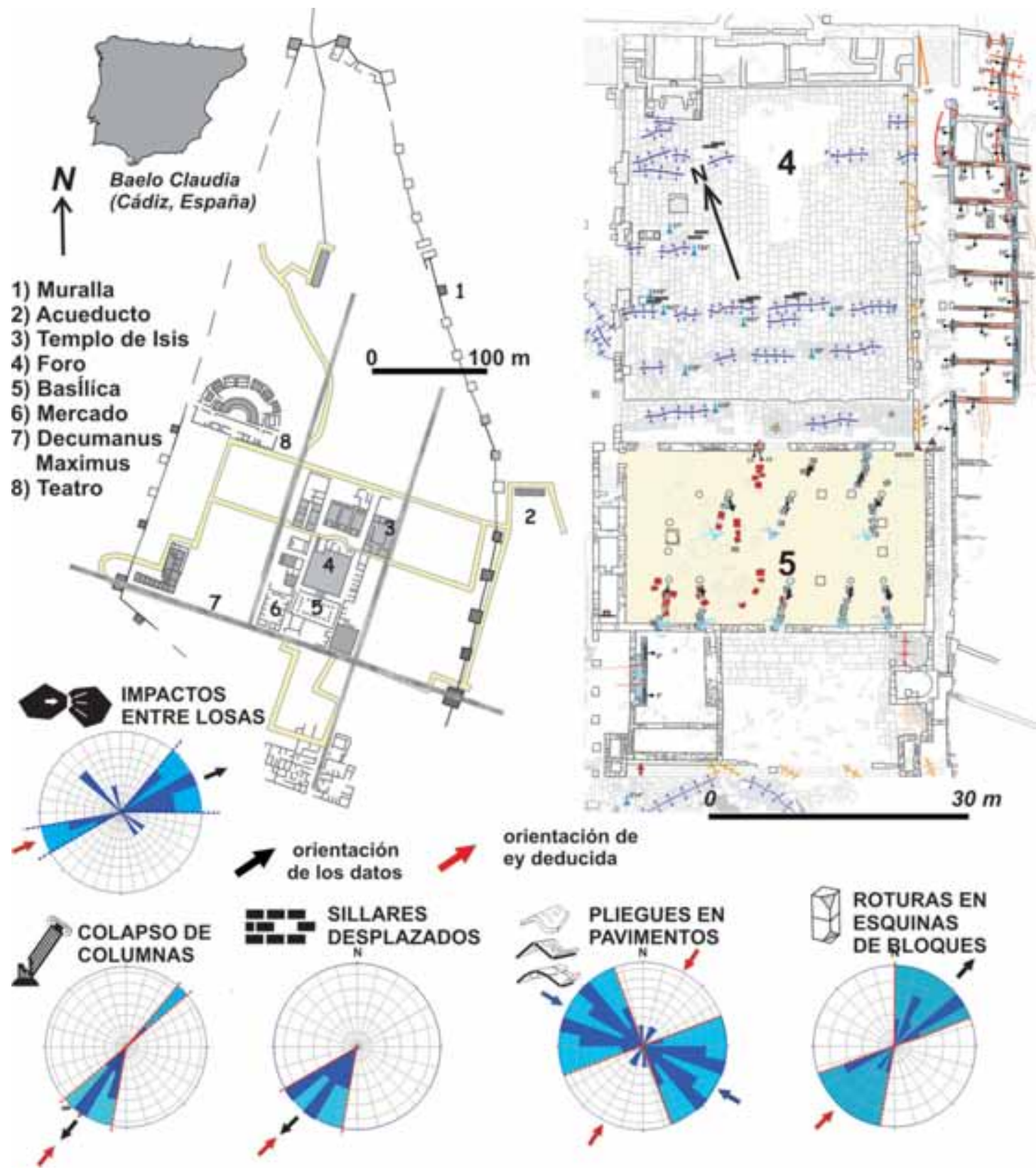

Figura 9. Mapa de la zona del Foro de Baelo Claudia (Cádiz, España) con los EAE inventariados (Silva et al., 2016). Resultados de varios tipos de efectos en la ciudad de Baelo Claudia: sillares desplazados, pliegues en pavimentos, impactos entre losas, colapso de columnas (caídas orientadas) y roturas en esquinas de bloques.

Figure 9. Map of the Baelo Claudia Forum (Cádiz, Spain) with the EAE inventory (Silva et al., 2016). The main types of effects recorded are: displaced masonry blocks, folds on pavements, shock breakouts in flagstones, fallen oriented columns and dipping broken corners. 
para mejorar la completitud de los catálogos sísmicos en países con altos periodos de recurrencia como España. En ese mismo ámbito, se está trabajando en otros yacimientos arqueológicos para poder establecer, a partir del análisis de datos arquesosimológicos, la posible ocurrencia de fenómenos sísmicos que no estén catalogados, como por ejemplo la posibilidad de un evento destructivo en el siglo IV afectara a la ciudad romana de Complutum (Madrid, España) (Rodríguez-Pascua et al., 2016) (Fig.10).

Esta metodología también se ha aplicado a terremotos históricos ampliamente documentados como el terremoto de Lisboa (Giner-Robles et al., 2009, Rodríguez-Pascua et al. 2011b), y en otros importantes terremotos históricos con menor información histórica como el terremoto de Estubeny (Valencia) de 1748 (Giner-Robles et al, 2014, Silva et al., 2014a), o el terremoto de Huercal-Overa (Almería) de 1863 (Silva et al., 2014b), ampliando el conocimiento de los efectos de estos eventos y por tanto mejorando futuros análisis de la peligrosidad sísmica.

\section{Calibración en terremotos instrumentales}

Para poder calibrar la metodología se han estudiado terremotos instrumentales analizando la orientación de los daños producidos por los eventos en edificaciones de todo tipo. En este tipo de eventos se sim-

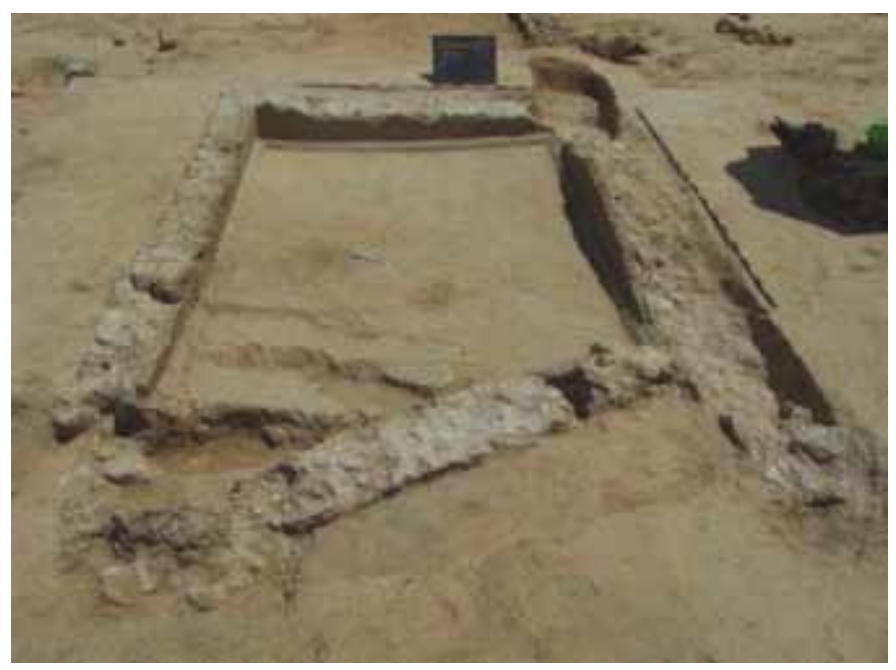

Figura 10. Cisterna de mortero (siglo IV A.D.) fracturada por una licuefacción del terreno (Rodríguez-Pascua et al., 2016) en las proximidades de la ciudad romana de Complutum (Madrid, España).

Figure 10. Mortar of the cistern (IV Century AD) fractured by a liquefaction process affecting the basement (Rodríguez-Pascua et al., 2016), located in the vicinity of the first setting corresponding to the Roman city of Complutum (Madrid, Spain). plifica el análisis, ya que únicamente se cuantifican las deformaciones sin necesidad de establecer la cronología de las mismas. Este tipo de calibración se ha realizado en dos terremotos instrumentales: el terremoto de Lorca (España) del 11 de mayo del 2011 (Mw $5,1)$, y en el terremoto de la Emilia Romagna (Italia) del 20 de mayo de 2012 (Mw 5,9).

En el terremoto de Lorca de 2011 se analizaron más de 144 daños orientados en la ciudad y sus alrededores, tanto en edificios históricos como en edificaciones normales (Giner-Robles et al., 2012a). Se inventariaron diferentes EAE (caídas orientadas, muros basculados, colapsados y plegados, dovelas de arco hundidas, desplazamientos de bloques y roturas en esquinas de bloques), estableciendo una orientación de daño principal según NO-SE (Fig 11) dirección coherente con la aceleración del suelo registrada en la ciudad de Lorca y con los parámetros focales del terremoto (Giner-Robles et al., 2012a).

También se analizaron diferentes daños en la colegiata de la ciudad, inventariando daños orientados muy similares en tipología, localización y orientación a los producidos por el terremoto de 1674 ocurrido en la misma ciudad (Rodríguez-Pascua et al., 2012), hecho que abre nuevas líneas de investigación en referencia al análisis y comparación de daños de diferentes terremotos para la definición de fuentes sísmica recurrentes.

Los terremotos de la Emilia Romagna (Italia) de 2012, supusieron una aportación importante en la calibración de la metodología de análisis ya que fueron afectados numerosos edificios históricos en diferentes ciudades de la región. El análisis de las estructuras con daños orientados (Pérez-López et al., 2012; Giner-Robles et al., 2013), ha permitido calibrar y definir adecuadamente la metodología de análisis de la deformación en diferentes tipos de efectos (e. g. caídas orientadas, desplazamientos de bloques,...) (Fig. 12).

\section{Discusión y conclusiones}

La metodología propuesta en este trabajo permite afrontar el análisis cuantificado de yacimientos arqueológicos susceptibles de haber sufrido daños por eventos sísmicos. Estos análisis se basan en la premisa de que los daños producidos por un terremoto en una localidad (o yacimiento arqueológico) siguen un patrón de orientaciones relacionado con las características de los parámetros focales del terremoto (localización, tipo de falla, directividad de la ruptura, etc.) (Giner-Robles et al., 2009, 2012; Howard et al., 2005; Motoki and Seo, 2000). Existen muchos 

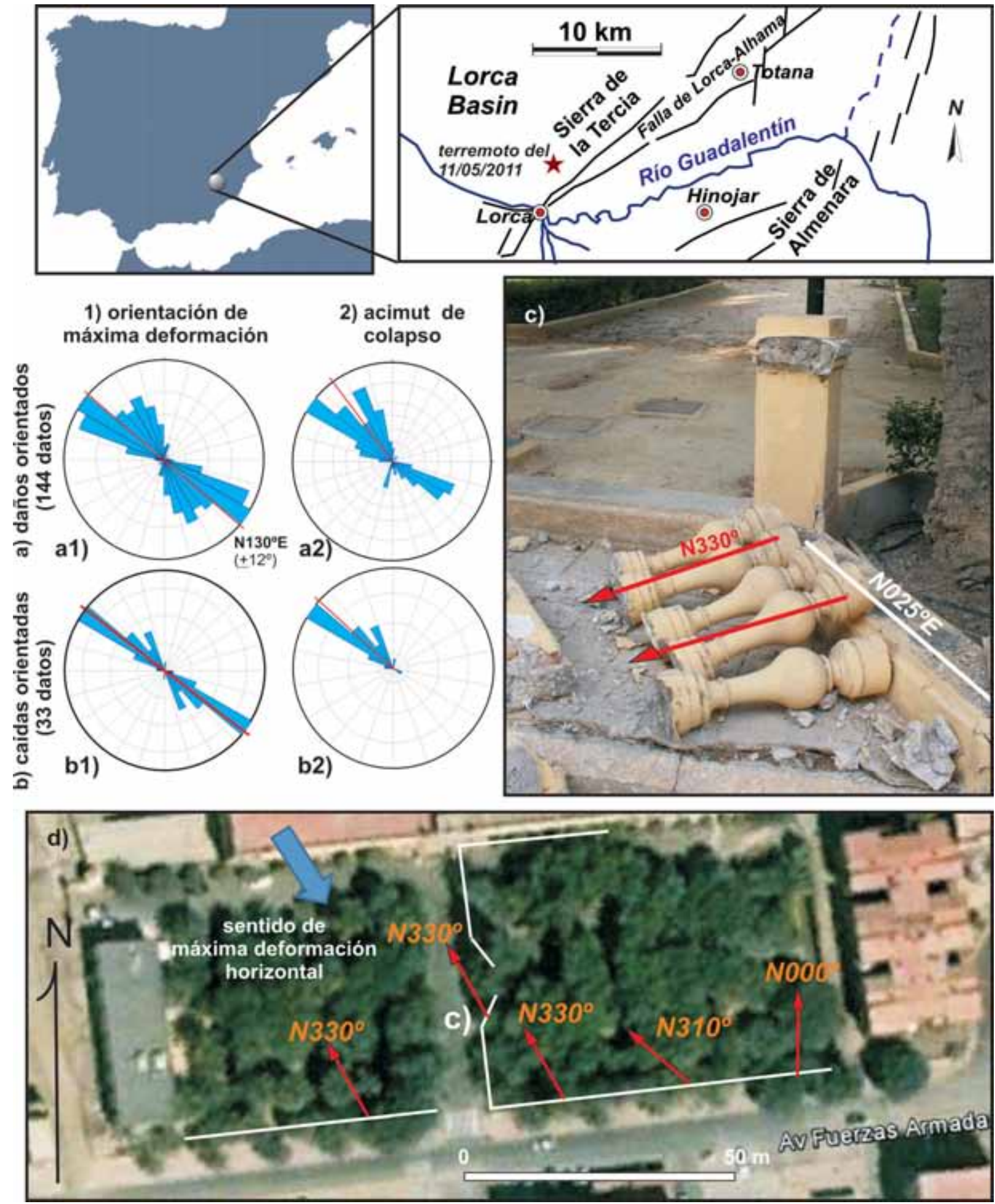

Figura 11. Daños orientados producidos por el terremoto de Lorca (Murcia, España) del 11 de mayo de 2011. Rosas de orientaciones de máxima deformación horizontal (1) y de azimut de caída (2), para: todos los daños registrados en la ciudad (a), y sólo de las estructuras de caídas orientados (b). c) Ejemplo de detalle de la caída orientada en la barandilla en un parque de la ciudad. d) Mapa del parque que ilustra la consistencia en las direcciones de deformación máxima y de acimut de caída (modificado de Giner-Robles et al., 2013).

Figure 11. Oriented damage analysis caused by the Lorca earthquake (Murcia, Spain) May 20, 2011. Rose diagram of the maximum deformation (1) and azimuth of the collapse structures (2), of all the data (a) and oriented fall structures (b). c) Detail of the fall on the balustrade in a city park. d) Map illustrating the consistency in the directions of maximum deformation and the azimuth of the main collapses (modified from Giner-Robles et al., 2013). 


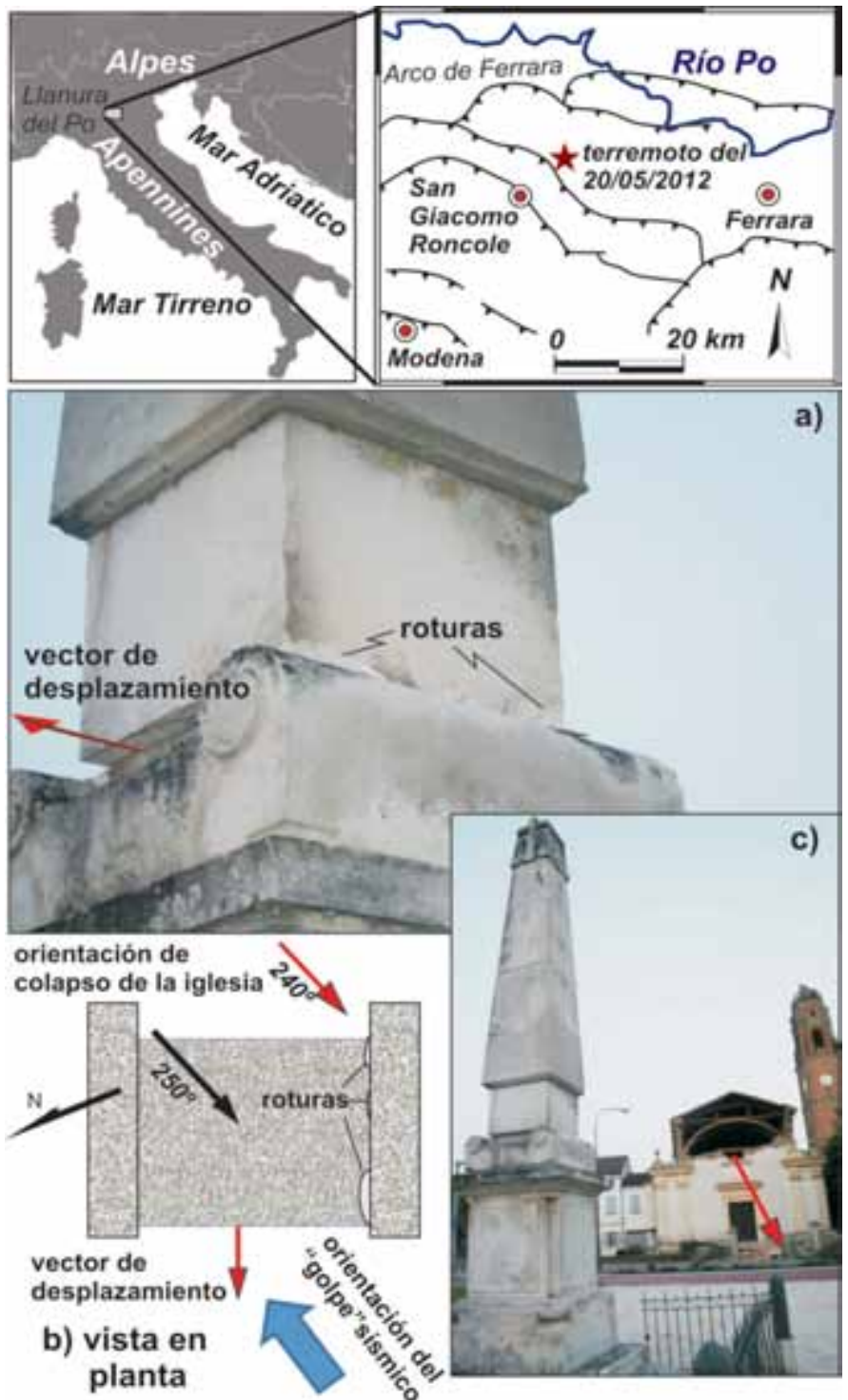

Figura 12. Ejemplo de análisis de EAE producidos por el terremoto de la Emilia Romagna (Italia) del 20 de mayo de 2012. Obelisco situado en el pueblo de San Giacomo Roncole (Emilia Romagna). El desplazamiento se produjo en la única dirección posible $(a, b)$, pero las grietas en el bloque indican que la dirección de la deformación máxima presenta una orientación subparalela al acimut de colapso de la fachada de la iglesia que si sitúa al fondo de la fotografía (c) (N240 ${ }^{\circ}$ (modificado de Giner-Robles et al., 2013).

Figure 12. Example of EAE analysis induced by the Emilia Romagna earthquake (Italy) on May 20, 2012. Obelisk in the village of San Giacomo Roncole. Displacement occurred in the only possible direction $(a, b)$, but cracks in the block indicate the direction of the maximum strain, sub-parallel to the collapse of the facade of the church in the background (c) $\left(\mathrm{N} 240^{\circ}\right)$ (modified from Giner-Robles et al., 2013).

trabajos que han afrontado el análisis de los daños producidos por terremotos en yacimientos arqueológicos y edificios históricos (e. g. Guidoboni, 1989;
Hancock and Stewart, 2001; Marco, 2008; Nikonov, 1988; Stiros and Jones, 1996), pero el principal problema era la cuantificación de los mismos. Esta metodología contempla el inventario (Rodriguez-Pascua et al., 2011b) y análisis de los daños mediante diferentes técnicas de caracterización de la deformación utilizadas en análisis clásicos de geología estructural (Giner-Robles et al., 2009, 2011). Estas técnicas nos permiten cuantificar la deformación a diferentes escalas de forma que podemos establecer, a partir de los patrones de orientación de los daños obtenidos, la homogeneidad de los resultados, y por tanto su posible origen sísmico.

La metodología se ha desarrollado de forma que los datos obtenidos puedan ser comparados tanto con escalas de intensidades clásicas (EMS o MSK) (Rodríguez-Pascua et al., 2013), como con escalas de intensidades basadas en los efectos geológicos de los terremotos (ESI-07, Michetti et al., 2007). De esta forma los datos pueden ser incorporados a los catálogos sísmicos y mejorar los análisis de peligrosidad sísmica.

\section{Agradecimientos}

Este trabajo ha sido financiado por los proyectos CGL2015-67169-P (QTECTSPAIN, USAL) y CATESI-07 (IGME). Es una contribución al INQUATERPRO Project 1299 y a los grupos de trabajo OTECT-AEQUA y GQMAEQUA.

\section{Referencias}

Giner-Robles, J.L., Rodríguez-Pascua, M.A., Pérez-López, R., Silva, P.G., Bardají, T., Grützner C. y Reicherter, K. 2009. Structural analysis of Earthquake Archaeological Effects (EAE) Baelo Claudia Examples (Cádiz, South Spain), UNED, Madrid, $137 \mathrm{pp}$.

Giner-Robles, J.L., Silva Barroso, P.G., Pérez-López, R., Rodríguez-Pascua, M.A., Bardají Azcárate, T., GarduñoMonroy, V.H. y Lario Gómez, J., 2011. Evaluación del daño sísmico en edificios históricos y yacimientos arqueológicos. Aplicación al estudio del riesgo sísmico. Proyecto EDASI. Serie Investigación. Fundación MAPFRE, Madrid. $96 \mathrm{pp}$.

Giner-Robles, J.L., Pérez-López, R., Rodríguez-Pascua, M.A., Silva Barroso, P.G., Martín-González, F. and Cabañas, L., 2012a. Oriented Structural analysis of seismically oriented damage caused by the Lorca earthquake of 11 May 2011: Application to archaeoseismology. Boletín Geológico y Minero, 123, 4, 503-513.

Giner-Robles J.L., Bardaji Azcárate T., Rodríguez-Pascua M. A., Pérez-López Raúl, Silva P.G., Garduño Monroy V. H. and Lario Gómez, J. 2012b. Postseismic earthquake 
archaeological effects (EAE s) in Morelia and Patzcuaro cities (Michoacan, Mexico). In: Garduño, V.H. and Rodríguez-Pascua, M.A. (eds.), Proceedings 3 INOUAIGCP 567 International Workshop on Active Tectonics, Paleoseismology and Archaeoseismology, Morelia, Mexico, 254-258.

Giner-Robles, J. L., Pérez-López, R., Rodríguez-Pascua, M.A., Silva, P.G., Martín-González, F. and Rodríguez-Escudero, E., 2013. A review on oriented fall structures (Earthquake Archaeological Effect, EAE) induced by instrumental earthquakes. Cuaternario y Geomorfología, 27 (3-4), 532.

Giner-Robles, J.L., Silva, P.G., Rodríguez-Pascua, M.A., Pérez-López, R. y Alberola Romá, A., 2014. In: ÁlvarezGómez, J.A. and Martín F. (eds.). Una aproximación multidisciplinar al estudio de las fallas activas, los terremotos y el riesgo sísmico. Instituto Geológico de España, Iberfault, 2014, 149-152.

Grützner; C., Reicherter, K., Hübscher, C. y Silva, P.G., 2012. Active faulting and neotectonics in the Baelo Claudia area, Campo de Gibraltar (Southern Spain). Tectonophysics, 554-557, 127-142.

Guidoboni, E., 1989. I terremoti prima del Mille in Italia e nell area Mediterranea: storia, archaeologia, sismología. Bologna. SGA-Instituto Nazionale di Geofisica. 765 pp.

Hancock, P.L. and Stewart, I.S., 2001. The Archaeology of Geological Catastrophes. Geological Society of London Special Publication, 171, 45-70.

Howard J.K., Tracy, C.A. y Burnsa R.G., 2005. Comparing observed and predicted directivity in near-source ground motion. Earthquake Spectra, 21 (4), pp.1063-1092.

Marco, S., 2008. Recognition of earthquake-related damage in archaeological sites: Examples from the Dead Sea fault zone. Tectonophysics, 453, 148-156.

Martinez-Solares, J.M., 2001. Los efectos en España del terremoto de Lisboa. Monografía $\mathrm{n}^{\circ} 19$. Dirección General del Instituto Geográfico Nacional, Madrid, 756 pp.

Michetti A. M., Esposito, E., Guerrieri, L., Porfido, S. E., Serva, L., Tatevossian, R., Vittori, E., Audemard, F., Asuma, T., Clague, J., Comerci, V., Gürpinar, A., McCalpin, J., Mörner, N.A., Ota, Y., Roghozin E. 2007. Intensity Scale ESI-2007. Memorie Descriptive Della Carta Geologica D'Italia, 74. APAT, SystemCart Srl, Roma, Italia.

Motoki, K. and Seo, K., 2000. Strong motion characteristics near the source region of the Hyogoken-Nanbu earthquake from analyses of the directions of structural failures. 12th World Conference on Earthquake Engineering. Paper 959, 6 pp.

Nikonov, A., 1988. On the metholology of archaeoseismic research into historical monuments. In: Marinos G.and G. Koukis, G. (eds). Engineering Geology of Ancient Works, Monuments and Historical Sites. Balkema, Roterdam. 1325-1320.

Pérez-López, R., Giner-Robles, J.L., Rodríguez-Pascua, Miguel A., Martín-González, F.and Silva, Pablo G., 2012. Discussing the seismogenic source for the Emilia Romagna seismic series (May 2012, Italy) from oriented damage and EAE s analysis. In: Proceedings 3rd INQUAIGCP-567 International Workshop on Active Tectonics,
Paleoseismology and Archaeoseismology, Morelia, Mexico. pp. 125-130.

Rodríguez-Pascua M.A., Silva, P.G., Perucha Atienza, M.A., J.L. Giner-Robles, J.L., Pérez-López, R., 2011 a. Earthquake Archaeological Effects generated by the Lisbon earthquake (first of November 1755) in the Coria s Cathedral (Cáceres, western Spain). En: 2nd INQUA-IGCP-567 International Workshop on Active Tectonics, Earthquake Geology, Archaeology and Engineering, Corinth, Greece, 204-206.

Rodríguez-Pascua, M.A., Pérez-López, R., Silva, P.G., GinerRobles, J.L., Garduño-Monroy, V.H. y Reicherter, K., 2011b. A Comprehensive Classification of Earthquake Archaeological Effects (EAE) for Archaeoseismology. Quaternary International, 242, 20-30.

Rodríguez-Pascua, M.A., Pérez-López, R., Giner-Robles, J.L., Silva, P.G., Garduño-Monroy, V.H. y Reicherter, K., 2012. Efectos Arqueológicos del terremoto de Lorca del 11 de mayo de 2011 en su Patrimonio Cultural. Nuevas estructuras de deformación y reactivación de estructuras anteriores pertenecientes al terremoto de 1674. Geo-Temas, 13.

Rodriguez-Pascua, M.A., Silva, P.G., Pérez-López, R., GinerRobles, J.L., Martín-González, F. y Perucha, M.A., 2013. Preliminary intensity correlation between macroseismic scales (ESI07 and EMS98) and Earthquake archaeological effects (EAEs). In: Grützner.C. and Reicherter C. (eds.). Seismic Hazard, Critical Facilities and Slow Active Faults. Proceedings of the 4th International INOUA Meeting on Paleoseismology, Active Tectonics and Archeoseismology. 133-137.

Rodríguez-Pascua M.A., Silva, P.G., Perucha, M.A., GinerRobles, J.L., Heras, C., Bastida, A.B., Carrasco, P., Roquero, E., Lario, J., Bardaji, T., Pérez-López, R. y Elez, J. (2016). Seismically induced liquefaction structures in La Magdalena archaeological site, the 4th century AD Roman Complutum (Madrid, Spain). Quaternary International, 334, 34-46.

Sillières, S., 1997. Baelo Claudia: Una ciudad Romana de la Bética. Junta de Andalucía- Casa de Velázquez, Madrid. 237 pp.

Silva, P.G., Borja, F., Zazo, C., Goy, J.L., Bardaji, T., De Luque, L., Lario J. y Dabrio, C.J., 2005. Archaeoseismic record at the ancient Roman City of Baelo Claudia (Cádiz, south Spain). Tectonophysics, 408 (1-4). 129-146.

Silva, P.G., Reicherter, K., Grutzner, CH., Bardaji, T., Lario, J., Goy, J.L., Zazo, C. y Becker-Heidmann, P., 2009. Surface and subsurface palaeoseismic records at the ancient Roman city of Baelo Claudia and the Bolonia Bay area, Cádiz (south Spain). Geological Society of London, Special Publication, 316. 93-121.

Silva P.G., Rodríguez-Pascua, M.A., Giner-Robles J. L., PérezLópez, R., Lario Gómez, J., Perucha Atienza, M.A., Bardají, T., Huerta, P., Roquero, E. y Bautista Davila, M.B., 2014a. Catálogo de Efectos Geológicos de los terremotos en España (Silva P.G. y Rodríguez-Pascua M.A. (eds.). Serie Riesgos Geológicos / Geotécnia IGME. Vol. 4. Instituto Geológico y Minero de España, Madrid, 350 pp.

Silva, P.G., Rodríguez-Pascua, M.A., Giner-Robles, J.L., Roquero, E., Pérez-López, R., Huerta, P. , Bardají, T., 2014b. Anatomy of an earthquake: geological analysis of the Huercal-Overa AD 1863 event (Almería, SE España). 
In: Álvarez-Gómez, J.A. and Martín F. (eds.). Una aproximación multidisciplinar al estudio de las fallas activas, los terremotos y el riesgo sísmico. Instituto Geológico de España, Iberfault, 2014, 145-148 pp.

Silva, P.G., Giner-Robles, J.L., Reicherter, K., RodríguezPascua, M.A., Grützner, C., García Jiménez, I., Carrasco García, P., Bardají, T., Santos, G., Roquero, E., Röth, J., Perucha, M.A., Pérez-López, R., Fernández Macarro, B.,
Martínez-Graña, A., Goy, J.L. y Zazo C. 2016. Ancient earthquakes in the Roman city of Baelo Claudia (Cádiz, South of Spain): Fifteen years of Archaeosimology research. Estudios Geológicos, 72 (1), 1-24

Stiros, S., Jones, R.E. 1996. Archaeoseismology. Institute of Geology and Mineral Exploration. Fitch Laboratory Occasional Paper. Stiros S., and Jones, R.E., Eds. Atenas. 268 p.

Recibido: marzo 2015

Revisado: mayo 2015

Aceptado: junio 2015

Publicado: marzo 2018 
